\title{
Comparison of visceral, body fat indices and anthropometric measures in relation to chronic kidney disease among Chinese adults from a large scale cross-sectional study
}

Ying Dong ${ }^{1}$, Zengwu Wang ${ }^{1 *}$, Zuo Chen ${ }^{1}$, Xin Wang ${ }^{1}$, Linfeng Zhang ${ }^{1}$, Jingyu Nie', Congyi Zheng ${ }^{1}$, Jiali Wang ${ }^{1}$, Lan Shao', Ye Tian ${ }^{1}$ and Runlin Gao ${ }^{2}$

\begin{abstract}
Background: The aim of the study was to assess the association between chronic kidney disease (CKD) and obesity in predicting CKD among Chinese adults, distinguishing between 5 different adiposity indices: visceral fat index (VFI), percentage body fat (PBF), body mass index (BMI), waist circumference (WC) and waist-to-height ratio (WHtR).

Methods: A total of 29,516 participants aged 35 years or above were selected using a stratified multistage random sampling method across China during 2012-2015. CKD was defined as an estimated glomerular filtration (eGFR) < $60 \mathrm{ml} / \mathrm{min} / 1.72 \mathrm{~m}^{2}$.
\end{abstract}

Results: The overall weighted prevalence of CKD was 3.94\% (3.62\% in males and $4.25 \%$ in females). All five adiposity indices had significant negative correlations to eGFR $(P<0.05)$. The area under the ROC (receiver operating characteristic) curves (AUC) for PBF was almost significantly larger than the other adiposity indices $(P<0$. 001). In addition, PBF yielded the highest Youden index in identifying CKD (male: 0.15; female: 0.20). In the logistic analysis, PBF had the highest crude odds ratios (ORs) in both males (OR: 1.819, 95\% Cl 1.559-2.123) and females (OR: 2.268, 95\% Cl 1.980-2.597). After adjusted for age, smoking status, alcohol use, education level, marital status, rural vs. urban area, geographic regions, and diagnosis of hypertension, diabetes mellitus, myocardial infarction and stroke, the ORs on PBF remained significant for both genders $(P<0.05)$.

Conclusions: Obesity is associated with an increased risk of CKD. Furthermore, PBF was a better predictor for identifying CKD than other adiposity indices (BMI, WC, WHtR, and VFI).

Keywords: Chronic kidney disease, Adiposity indices, Percentage body fat, Chinese adults, Cross-sectional study

\section{Background}

Chronic kidney disease (CKD) has been a major global health problem. It also plays an important role in development of end-stage renal disease (ESRD) [1], all-cause mortality [2], and non-vascular health outcomes [3]. Currently, CKD is the 12th highest cause of death (14\%

\footnotetext{
* Correspondence: wangzengwu@foxmail.com

${ }^{1}$ Division of Prevention and Community Health, National Center for Cardiovascular Disease, Fuwai Hospital, Pecking Union Medical College \&

Chinese Academy of Medical Sciences, Beijing 102308, China

Full list of author information is available at the end of the article
}

of all deaths) worldwide [4]. A large representative survey in the United States has shown that the prevalence of CKD among adults aged $\geq 45$ years' was $11.6 \%$ [5]. In China, the overall prevalence of CKD among adults aged 18 years or older was $10.8 \%$ [6].

Obesity is becoming a global health concern. Over the past decade, changes in dietary and physical activity patterns has led to an increase in obesity prevalence, especially in large cities [7]. Previous studies have shown that the prevalence of obesity increased from $4.0 \%$ in 1993 to $10.7 \%$ in 2009 among Chinese adults [8]. An increasing 
body of evidence suggests that obesity, a risk factor of kidney disease, had a direct impact on the development of CKD and end stage renal disease (ESRD) [9]. Among many indicators of obesity, body mass index (BMI) and waist circumference (WC) have been widely used to define general and abdominal obesity, respectively [10]. However, BMI cannot differentiate between lean and fat mass, and WC does not account for the effect of height on risk [11]. Waist-to-height ratio (WHtR) was introduced as an alternative to WC. Additional obesity indicators include percentage body fat (PBF), which is defined as the percentage of individual fat mass over body weight [12]; and visceral fat index (VFI), an accurate and reliable indicator for evaluating body fat stored around important organs, such as the liver, intestines and pancreas [11].

The identification of CKD risk factors is essential, especially in the face of an increasing prevalence of CKD and obesity. Currently, it is unknown which of the existing adiposity indices is best for predicting CKD. Previous studies have only considered one or two types of adiposity measurement in relation to CKD [13-15]. Thus, we conducted this cross-sectional study in Chinese adults to assess the association between obesity and $C K D$, and compared the performance of five adiposity indices (BMI, WC, WHtR, PBF and VFI) to predict CKD.

\section{Methods}

\section{Study population}

This cross-sectional investigation was conducted from 2012 to 2015. This cross-sectional study was a part of a 2012-2015 survey on the prevalence of hypertension in China, spanning 31 provinces with 262 cities/counties; a detailed description of sampling methods has been previously published [13]. The 262 cities and counties were stratified into Eastern, Middle and Western regions, based on economic development status. Using simple random sampling (SRS), 16 cities and 17 counties were selected for this study, including 7 cities and 7 counties in the Eastern region, 6 cities and 6 counties in the Middle region, and 3 cities and 4 counties in the Western region. To meet the designed sample size of 35,000 participants aged $\geq 35$ years and take non-response into account in the survey 56,000 subjects were randomly selected from the eligible sites. As a result, 34,994 participants completed the survey, the overall response rate was $62.5 \%$. After excluded the subjects without blood pressure $(n=414)$, demographic information $(n=485)$, adiposity indices $(n=1836)$, laboratory test values $(n=$ 2743), 29,516 were included in the final analysis. All the participants gave written informed consent prior to data collection. The ethical review committee of Fuwai Hospital (Beijing, China) and each participating center (Additional file 1) approved the study's protocol.

\section{Adiposity indices}

All staff involved in the study were trained and certified prior to survey implementation, according to a uniform protocol and operation manual. Height was measured to the nearest $5 \mathrm{~mm}$ with a standard stadiometer without shoes in the standing position. WC was measured to the nearest $5 \mathrm{~mm}$ directly touching the participant's skin using a cloth tape. Weight was measured to the nearest $0.1 \mathrm{~kg}$ with light clothing and no shoes. PBF and VFI were measured using an OMRON body fat and weight measurement device (V-body HBF-371, OMRON, Kyoto, Japan). BMI was calculated as weight (in kilograms) divided by the square of height (in meters). WHtR was calculated as $\mathrm{WC}$ in centimeters divided by height in centimeters.

\section{Glomerular filtration rate assessment}

Fasting blood samples were collected in the morning after 10-12 $\mathrm{h}$ fasting. The samples were processed properly and refrigerated immediately. All samples were analyzed in a designated central laboratory (Beijing Adicon Clinical Laboratories, INC, Beijing, China). The serum creatinine was measured using Jaffe's kinetic method. Estimated glomerular filtration rate (eGFR) was calculated using the equation originating from the modified Modification of Diet in Renal Disease formula for Chinese patients [14]: eGFR $=175^{*} \mathrm{Scr}^{-1.234 *}$ age $^{-0.179}$ [if female, *0.79], where Scr is serum creatinine concentration $(\mathrm{mg} / \mathrm{dL})$ and age is in years. CKD was defined as eGFR $<60 \mathrm{ml} / \mathrm{min} / 1.73 \mathrm{~m}^{2}$.

\section{Covariate measurements}

Eligible participants completed the standardized questionnaire through face-to-face interview by trained staff to obtain information on demographic characteristics, lifestyle factors (i.e. smoking and alcohol use) and comorbidities (hypertension, diabetes mellitus, myocardial infarction and stroke). Smoking status was defined as a person who smoked in the past month or total cigarette consumption more than 20 packages in the lifetime. Alcohol use was defined as a person who consumed more than one alcoholic drink per week in the last 30 days. Hypertension was defined as systolic blood pressure (SBP) $\geq 140 \mathrm{mmHg}$, and/or diastolic blood pressure (DBP) $\geq 90 \mathrm{mmHg}$, and/ or self-reported history of antihypertensive medications for treatment of hypertension in the last 2 weeks. Diabetes mellitus was defined as participants with previously diagnosed diabetes and used insulin or hypoglycemic agents, or had a fasting blood glucose $\geq 7.0 \mathrm{mmol} / \mathrm{L}$.

\section{Statistical analysis}

Continuous variables were presented as means \pm standard deviations (SDs) and compared using the Student's $t$-test or Wilcoxon rank-test. Categorical variables were presented as numbers (percentages) and compared using Chi-square test. The Pearson correlation coefficient was used to assess 
the correlation between each of the adiposity indices (BMI, WC, WHtR, PBF and VFI) and eGFR.

Receiver operating characteristics curve (ROC) analyses for both genders were used to determine the optimal cutoff values of each adiposity index for CKD with the maximum Youden index (sensitivity+specificity-1). Additionally, the areas under the ROC curve (AUCs) of these five adiposity indices were used to determine their reliability as predictive markers of CKD; statistical significance determined by applying the method of DeLong et al. [15] using MedCalc version 11.4.2.0.

Each adiposity index was divided into lower and higher level groups according to optimal gender- specific cut-off values performed in following ROC analyses. The association between the adiposity and CKD in both genders was calculated using multiple logistic regression analyses after adjusting age, education level, marital status, areas (rural/urban), region (eastern, middle and western region), smoking status, alcohol use, hypertension, diabetes mellitus, myocardial infarction and stroke.

The data analyses were performed using SAS version 9.3 (SAS Institute Inc., Cary, North Carolina, USA). Sample weights were adjusted for non-response, total population (according to 2010 Chinese census) and region to obtain Chinese nationally representative prevalence estimates. All $P$ values were two-sided, $P<0.05$ was considered statistically significant.

\section{Results}

A total of 29,516 participants $(13,410$ males, and 16,106 females) were included in this study with a mean age of $56.48 \pm 13.13$ years. The overall weighted prevalence of CKD was $3.94 \%$ (3.62\% in male and $4.25 \%$ in female).

Table 1 presents the characteristics of individuals included in the current analysis. The prevalence of CKD in both male and female tended to increase with age $(P$ for

Table 1 Baseline characteristics of study population

\begin{tabular}{|c|c|c|c|c|c|c|c|}
\hline & \multicolumn{3}{|c|}{ Male $(n=13,410)$} & \multicolumn{3}{|c|}{ Female $(n=16,106)$} & \multirow[t]{2}{*}{ Total $(n=29,516)$} \\
\hline & $\mathrm{eGFR} \geq 60$ & eGFR<60 & $P$ & $e G F R \geq 60$ & eGFR<60 & $P$ & \\
\hline \multicolumn{8}{|l|}{ Age (year) } \\
\hline $35 \sim 44$ & $2942(23.11)$ & $17(2.49)$ & $<0.0001$ & $3953(25.99)$ & $23(2.57)$ & $<0.0001$ & $6935(23.50)$ \\
\hline $45 \sim 54$ & $3001(23.58)$ & $40(5.87)$ & & $3950(25.97)$ & $61(6.82)$ & & $7052(23.89)$ \\
\hline $55 \sim 64$ & $2938(23.08)$ & $95(13.93)$ & & $3453(22.70)$ & $144(16.11)$ & & $6630(22.46)$ \\
\hline $65 \sim 74$ & $2476(19.45)$ & 237 (34.75) & & $2566(16.87)$ & $295(33.00)$ & & $5574(18.88)$ \\
\hline$\geq 75$ & $1371(10.77)$ & $293(42.96)$ & & $1290(8.48)$ & $371(41.50)$ & & $3325(11.27)$ \\
\hline Urban & $5870(46.12)$ & $305(44.72)$ & 0.4760 & $6636(43.62)$ & $405(45.30)$ & 0.3250 & $13,216(44.78)$ \\
\hline \multicolumn{8}{|l|}{ Region } \\
\hline Eastern & $5464(42.93)$ & $302(44.28)$ & $<0.0001$ & $6549(43.05)$ & 339 (37.92) & $<0.0001$ & $12,654(42.87)$ \\
\hline Middle & $5200(40.85)$ & $220(32.26)$ & & $5814(38.22)$ & $283(31.66)$ & & $11,517(39.02)$ \\
\hline Western & $2064(16.22)$ & $160(23.46)$ & & $2849(18.73)$ & $272(30.43)$ & & $5345(18.11)$ \\
\hline Higher Education (> 12 years) & $957(7.52)$ & $46(6.74)$ & 0.4540 & $847(5.57)$ & $24(2.68)$ & $<0.0001$ & $1874(6.35)$ \\
\hline Married & $11,640(91.47)$ & $547(80.21)$ & $<0.0001$ & $13,263(87.19)$ & $565(63.20)$ & $<0.0001$ & $26,015(88.14)$ \\
\hline Smoking status & $5861(46.05)$ & $226(33.14)$ & $<0.0001$ & $516(3.39)$ & $42(4.70)$ & 0.0380 & $6645(22.51)$ \\
\hline Alcohol use & $4946(38.86)$ & $157(23.02)$ & $<0.0001$ & $541(3.56)$ & $28(3.13)$ & 0.5040 & $5672(19.22)$ \\
\hline Hypertension & $5184(40.73)$ & $440(64.52)$ & $<0.0001$ & $5603(36.83)$ & $589(65.88)$ & $<0.0001$ & $11,816(40.03)$ \\
\hline DM & $1281(10.06)$ & $114(16.72)$ & $<0.0001$ & $1298(8.53)$ & $152(17.00)$ & $<0.0001$ & $2845(9.64)$ \\
\hline Ml & $97(0.76)$ & $17(2.49)$ & $<0.0001$ & $93(0.61)$ & $15(1.68)$ & $<0.0001$ & $222(0.75)$ \\
\hline Stroke & $340(2.67)$ & $54(7.92)$ & $<0.0001$ & $266(1.75)$ & $40(4.47)$ & $<0.0001$ & $700(2.37)$ \\
\hline Weight (kg) & $67.61 \pm 11.19$ & $65.65 \pm 11.90$ & $<0.0001$ & $59.45 \pm 9.99$ & $57.83 \pm 10.78$ & $<0.0001$ & $63.05 \pm 11.36$ \\
\hline BMI $\left(\mathrm{kg} / \mathrm{m}^{2}\right)$ & $24.40 \pm 3.36$ & $24.28 \pm 3.64$ & 0.3585 & $24.70 \pm 3.64$ & $24.80 \pm 4.03$ & 0.4103 & $24.56 \pm 3.53$ \\
\hline$W C(\mathrm{~cm})$ & $85.43 \pm 9.93$ & $85.73 \pm 10.77$ & 0.4479 & $82.31 \pm 10.04$ & $84.75 \pm 11.34$ & $<0.0001$ & $83.81 \pm 10.17$ \\
\hline WHtR & $0.51 \pm 0.06$ & $0.52 \pm 0.06$ & 0.0003 & $0.53 \pm 0.07$ & $0.56 \pm 0.07$ & $<0.0001$ & $0.52 \pm 0.06$ \\
\hline PBF & $24.92 \pm 5.34$ & $26.59 \pm 5.51$ & $<0.0001$ & $33.25 \pm 5.23$ & $35.16 \pm 5.78$ & $<0.0001$ & $29.56 \pm 6.75$ \\
\hline VFI & $11.04 \pm 4.79$ & $12.02 \pm 5.59$ & $<0.0001$ & $7.87 \pm 4.08$ & $9.12 \pm 4.86$ & $<0.0001$ & $9.37 \pm 4.74$ \\
\hline
\end{tabular}

eGFR estimated glomerular filtration rate, the unit was as $\mathrm{ml} / \mathrm{min} / 1.73 \mathrm{~m}^{2}, B M I$ body mass index, WC waist circumference, $W H t R$ waist-to-height ratio, $P B F$ percent body fat, VFI visceral fat index, DM diabetes mellitus, $M I$ myocardial infarction

Data are expressed as the mean \pm SD or as $n(\%)$ 
trend < 0.001). Furthermore, we found that participants who had hypertension, diabetes mellitus, myocardial infarction or stroke had a higher risk of CKD in both genders (all $P<0.001)$. In addition, the values of BMI, WC, WHtR, PBF, and VFI almost consistently higher among participants with CKD, while only WHtR, PBF and VFI had statistical significant in both genders (all $P<0.001$ ).

Pearson's correlation coefficients for the 5 adiposity indices each against eGFR stratified by gender are illustrated in Table 2. We found that all these indices had a significant negative correlation to eGFR $(P<0.0001)$. BMI had the lowest Pearson's correlation coefficient in both genders (Pearson's correlation coefficient:- 0.051 in males; - 0.014 in females) which indicated the weakest correlations with eGFR.

Figure 1 shows the AUC values (95\% confidence interval $(\mathrm{CI})$ and Youden index for each of the five adiposity indices in prediction of CKD. PBF had a significantly higher AUC in both male and female groups (AUC for males: 0.593, 95\% CI: 0.584-0.601; AUC for females: 0.617, 95\% CI: 0.609-0.624) than the other indices (with WHtR in females being an exception). PBF also yielded the highest Youden index in identifying CKD (male: 0.15; female: 0.20).

Odds ratios (ORs) for the association between the CKD and high levels of various adiposity indices stratified by gender are shown in Table 3. PBF had the highest crude OR for CKD in male [1.819 (1.559-2.123)] and in female groups [2.268 (1.980-2.597)]. After adjusted for confounding factors, the ORs on PBF were also significant for both genders $(P<0.01)$. Table 4 shows the performance of the five adiposity indices according to Z-score standardization stratified by gender. As shown, after adjusted for confounding factors, a 1-SD increment change for each adiposity index was associated with higher risk of CKD in both male and female (all $P<0.05$ ). PBF had the highest ORs for CKD in both genders.

\section{Discussion}

The prevalence of CKD $\left(e G F R<60 \mathrm{ml} / \mathrm{min} / 1.73 \mathrm{~m}^{2}\right)$ in middle-aged Chinese adults was $3.94 \%$ (3.62\% in male and $4.25 \%$ in female). All of the adiposity indices (BMI,

Table 2 Pearson's correlation coefficients between adiposity indices and eGFR

\begin{tabular}{lllll}
\hline & eGFR & & & \\
\cline { 2 - 5 } & Male & $P$ & Female & $P$ \\
\hline BMI $\left(\mathrm{kg} / \mathrm{m}^{2}\right)$ & -0.051 & $<0.0001$ & -0.014 & $<0.0001$ \\
WC $(\mathrm{cm})$ & -0.061 & $<0.0001$ & -0.070 & $<0.0001$ \\
WHtR & -0.069 & $<0.0001$ & -0.097 & $<0.0001$ \\
PBF & -0.129 & $<0.0001$ & -0.151 & $<0.0001$ \\
VFI & -0.139 & $<0.0001$ & -0.112 & $<0.0001$ \\
\hline
\end{tabular}

eGFR estimated glomerular filtration rate, $B M I$ body mass index, WC waist circumference, $W H t R$ waist-to-height ratio, $P B F$ percent body fat, VFI visceral fat index
WC, WHtR, PBF and VFI) had a significant negative correlation to CKD in both genders. Obesity is associated with an increased risk of CKD. For the comparison, we found that PBF was a more suitable screening tool for predicting CKD than other adiposity indices for CKD in both males and females.

Several cross-sectional studies demonstrated that the prevalence of CKD differed substantially between geographic regions in China. The prevalence of CKD $\left(\mathrm{eGFR}<60 \mathrm{ml} / \mathrm{min} / 1.73 \mathrm{~m}^{2}\right)$ in southern China was $3.2 \%$ ( $2.2 \%$ in male vs. $4.1 \%$ in female) [16] which was a little lower than the results in our study. While, in the study conducted in Beijing among participants older than 40 years found that the prevalence of reduced renal function $\left(\mathrm{eGFR}<60 \mathrm{ml} / \mathrm{min} / 1.73 \mathrm{~m}^{2}\right)$ was $5.2 \%$ [17], which was higher than our present study. In addition to methodology, the heterogeneity might be related to differences in lifestyles, medical care and socioeconomic characteristics in different regions of China [18].

Previously, the majority of existing literature has focused on the relationship between BMI and/or WC with CKD. A cross-sectional study conducted in Chinese adults showed that for each increase of $1.0 \mathrm{~kg} / \mathrm{m}^{2}$ in BMI, a decline of $0.5 \mathrm{ml} / \mathrm{min} / 1.73 \mathrm{~m}^{2}$ [19]. In addition, a prospective cohort study, which included 827 incident cases of ESRD occurred during an average follow-up of 15.5 years, showed that patients had more than 1.1 times higher risk for developing ESRD participants with a higher BMI (BMI $\left.\geq 27.5 \mathrm{~kg} / \mathrm{m}^{2}\right)$, compared to a normal BMI (18.5 to $\left.23 \mathrm{~kg} / \mathrm{m}^{2}\right)$ [20]. Furthermore, a 7-year population-based cohort study conducted in Iran indicated that, irrespective of general obesity, the risk of developing CKD rose with increasing WC [21]. Even though BMI and WC have been the most widely used measurements to classify obesity, BMI does not account for fat distribution [22]; and WC does not account for height [11]. Thus, other indices which can more accurately measure total body fat quantity and visceral fat, such as WHtR, PBF and VFI, were developed. In our study, we found that WHtR, PBF and VFI correlated much more strongly with eGFR than BMI and WC (according to Pearson's correlation coefficients in Table 2).

A related study conducted among female subjects aged 65-80 years found that WHtR was a better index associated with CKD when comparing with other common adiposity indices (BMI, WC, waist-to-hip ratio) [23]. Dai et al. suggested that a visceral adiposity index was superior to BMI and WC for predicting CKD in females [24]. Additionally, a cross-sectional study [25] conducted in Chinese adults indicated that body fat percentage was significantly related to increased risk of CKD (OR: 1.049, 95\%CI: 1.006-1.093). Our study found that PBF had the highest AUC in both male and female groups. After adjusting for confounding factors, PBF still had the highest ORs for CKD than other adiposity indices in both genders. 

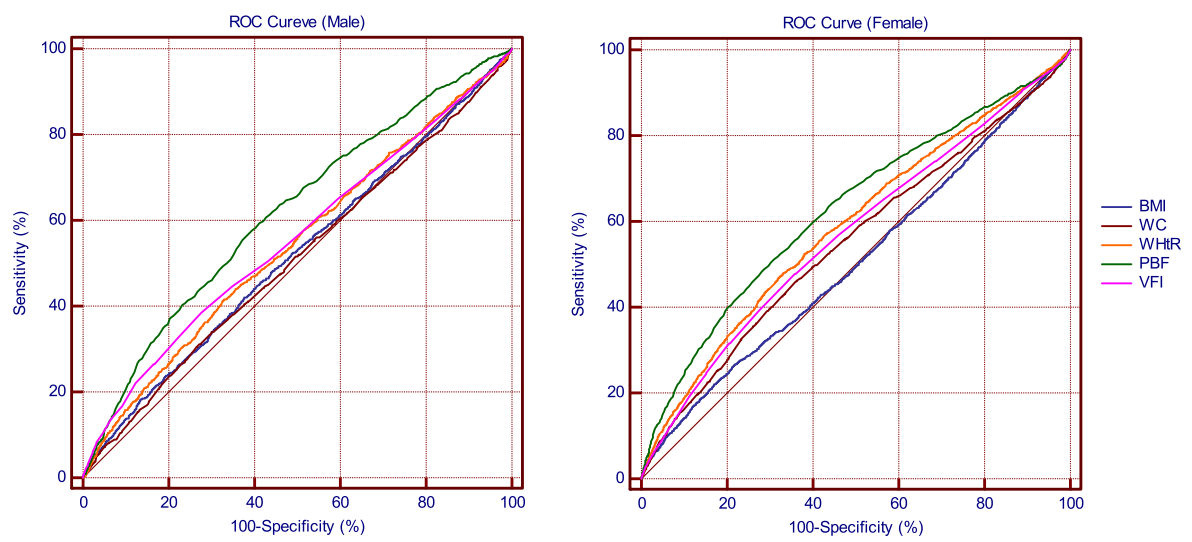

\begin{tabular}{lccccc}
\hline & AUC & $95 \%$ CI & Sens/Spec (\%) & Youden index & Cut-off \\
\hline BMI & $0.508^{\mathrm{b}}$ & $0.500-0.517$ & $18.91 / 86.86$ & 0.06 & 20.62 \\
WC & $0.513^{\mathrm{b}}$ & $0.505-0.522$ & $35.34 / 69.69$ & 0.05 & 90.20 \\
WHtR & $0.542^{\mathrm{b}}$ & $0.534-0.551$ & $44.57 / 64.22$ & 0.09 & 0.53 \\
PBF & 0.593 & $0.584-0.601$ & $51.32 / 63.43$ & 0.15 & 26.90 \\
VFI & $0.548^{\mathrm{b}}$ & $0.540-0.556$ & $37.83 / 71.81$ & 0.10 & 13.60 \\
\hline
\end{tabular}

\begin{tabular}{cccccc}
\hline & AUC & $95 \%$ CI & Sens/Spec (\%) & Youden index & Cut-off \\
\hline BMI & $0.511^{\mathrm{b}}$ & $0.503-0.518$ & $49.55 / 55.09$ & 0.05 & 24.88 \\
WC & $0.569^{\mathrm{b}}$ & $0.561-0.577$ & $46.20 / 66.01$ & 0.12 & 86.00 \\
WHtR & 0.601 & $0.593-0.608$ & $49.66 / 66.76$ & 0.16 & 0.56 \\
PBF & 0.617 & $0.609-0.624$ & $52.91 / 67.14$ & 0.20 & 35.70 \\
VFI & $0.577^{\mathrm{b}}$ & $0.569-0.584$ & $41.95 / 71.65$ & 0.14 & 9.60 \\
\hline
\end{tabular}

Fig. 1 AUC of adiposity indices to identifying subjects with chronic kidney disease according to sex. Sens: sensitivity; Spec: specificity; ROC curve: receiver operating characteristics curve; AUC: area under the ROC curve; BMl: body mass index; WC: waist circumference; WHtR: waist-to-height ratio; PBF: percentage body fat; VFI: visceral fat index

There are a few mechanisms that can explain the association between obesity and renal injury, including hemodynamic effects, inflammation and renal lipotoxicity [26]. Angiotensin II is elevated in the blood of obese individuals, leading to vasoconstriction of efferent arterioles; plasma aldosterone is also slightly elevated in obese individuals, causing salt and water retention, and ultimately proteinuria $[26,27]$. In regarding to inflammation, cytokines such as interleukin-6 (IL-6) and tumor necrosis factor - alpha (TNF- $\alpha$ ) play a major role in CKD development. IL-6 invades the adipose tissue and influences transforming growth factor beta 1 (TGF- $\beta 1$ ) receptor trafficking, leading to renal fibrosis [28]. TNF- $\alpha$ inhibits the activity of the nephron gene promoter in cultured podocytes, resulting in podocyte dysfunction in the obese population $[29,30]$.

Our study has several strengths. Firstly, we enrolled a large population-based sample, including male and female participants from China. Secondly, to our knowledge, this is the first time a comparison across five adiposity indices

Table 3 Odds ratios and 95\% confidence interval for chronic kidney disease according to adiposity indices stratified by gender

\begin{tabular}{lllr}
\hline & Model 1 & Model 2 & Model 3 \\
\hline Male & & & $0.954(0.768-1.186)$ \\
BMI & $0.650(0.533-0.792)^{\mathrm{b}}$ & $1.061(0.857-1.314)$ & $1.445(1.205-1.731)^{\mathrm{b}}$ \\
WC & $1.248(1.062-1.466)^{\mathrm{b}}$ & $1.617(1.355-1.929)^{\mathrm{b}}$ & $1.295(1.092-1.535)^{\mathrm{b}}$ \\
WHtR & $1.357(1.163-1.584)^{\mathrm{b}}$ & $1.446(1.225-1.708)^{\mathrm{b}}$ & $1.409(1.197-1.657)^{\mathrm{b}}$ \\
PBF & $1.819(1.559-2.123)^{\mathrm{b}}$ & $1.728(1.476-2.024)^{\mathrm{b}}$ & $1.357(1.138-1.617)^{\mathrm{b}}$ \\
VIF & $1.549(1.321-1.817)^{\mathrm{b}}$ & $1.519(1.279-1.804)^{\mathrm{b}}$ & $1.301(1.121-1.511)^{\mathrm{b}}$ \\
Female & $1.196(1.045-1.368)^{\mathrm{b}}$ & & $1.262(1.087-1.464)^{\mathrm{b}}$ \\
BMI & $1.647(1.439-1.886)^{\mathrm{b}}$ & $1.390(1.202-1.609)^{\mathrm{b}}$ & $1.188(1.025-1.378)^{\mathrm{a}}$ \\
WC & $1.961(1.713-2.246)^{\mathrm{b}}$ & $1.303(1.127-1.507)^{\mathrm{b}}$ & $1.520(1.315-1.757)^{\mathrm{b}}$ \\
WHtR & $2.268(1.980-2.597)^{\mathrm{b}}$ & $1.956(1.701-2.249)^{\mathrm{b}}$ & $1.284(1.105-1.493)^{\mathrm{b}}$ \\
PBF & $1.824(1.589-2.092)^{\mathrm{b}}$ & $1.422(1.227-1.648)^{\mathrm{b}}$ & $1.660 \mathrm{~b}$ \\
VIF & & & 1.239 \\
\hline
\end{tabular}

$B M I$ body mass index, WC waist circumference, $W H t R$ waist-to-height ratio, $P B F$ percent body fat, $V F I$ visceral fat index

Data are expressed as the odds ratios (95\% confidence interval)

Model 1: Non-adjusted

Model 2: Adjusted for age, smoking status, alcohol use, education level, marital status, living area (rural/urban) and region (eastern, middle and western)

Model 3: Adjusted for age, smoking status, alcohol use, education level, marital status, living area (rural/urban), region (eastern, middle and western),

hypertension, diabetes mellitus, myocardial infarction and stroke

${ }^{\mathrm{a}} P<0.05,{ }^{\mathrm{b}} P<0.01$ 
Table 4 Standardized odds ratios and 95\% confidence interval for chronic kidney disease according to adiposity indices stratified by gender

\begin{tabular}{|c|c|c|c|}
\hline & Model 1 & Model 2 & Model 3 \\
\hline \multicolumn{4}{|l|}{ Male } \\
\hline BMI z-score & $0.964(0.892-1.042)$ & $1.241(1.143-1.347)^{b}$ & $1.163(1.068-1.266)^{b}$ \\
\hline WC z-score & $1.030(0.954-1.113)$ & $1.203(1.109-1.305)^{\mathrm{b}}$ & $1.128(1.037-1.226)^{b}$ \\
\hline WHtR z-score & $1.153(1.067-1.245)^{b}$ & $1.175(1.085-1.272)^{b}$ & $1.103(1.016-1.197)^{\mathrm{a}}$ \\
\hline PBF z-score & $1.376(1.272-1.488)^{b}$ & $1.329(1.227-1.439)^{b}$ & $1.186(1.091-1.289)$ \\
\hline VIF z-score & $1.216(1.129-1.309)^{b}$ & $1.215(1.127-1.309)^{b}$ & $1.145(1.059-1.237)^{b}$ \\
\hline \multicolumn{4}{|l|}{ Female } \\
\hline BMI z-score & $1.029(0.962-1.100)$ & $1.170(1.092-1.253)^{\mathrm{b}}$ & $1.108(1.032-1.190)^{\mathrm{b}}$ \\
\hline WC z-score & $1.267(1.185-1.354)^{\mathrm{b}}$ & $1.194(1.116-1.278)^{b}$ & $1.138(1.062-1.220)^{b}$ \\
\hline WHtR z-score & $1.423(1.332-1.519)^{b}$ & $1.146(1.071-1.227)^{b}$ & $1.093(1.020-1.172)^{a}$ \\
\hline PBFz-score & $1.485(1.380-1.599)^{\mathrm{b}}$ & $1.337(1.242-1.441)^{b}$ & $1.153(1.070-1.244)^{b}$ \\
\hline VIF z-score & $1.302(1.227-1.381)^{b}$ & $1.167(1.094-1.244)^{b}$ & $1.108(1.036-1.185)^{b}$ \\
\hline
\end{tabular}

$B M I$ body mass index, WC waist circumference, $W H t R$ waist-to-height ratio, $P B F$ percent body fat, $V F I$ visceral fat index

Data are expressed as the odds ratios (95\% confidence interval)

Model 1: Non-adjusted

Model 2: Adjusted for age, smoking status, alcohol use, education level, marital status, living area (rural/urban) and region (Eastern, middle and Western)

Model 3: Adjusted for age, smoking status, alcohol use, education level, marital status, living area (rural/urban), region (Eastern, middle and Western),

hypertension, diabetes mellitus, myocardial infarction and stroke

${ }^{\mathrm{a}} P<0.05,{ }^{\mathrm{b}} P<0.01$

(BMI, WC, WHtR, PBF, VFI) has been made to predicting CKD in a large Chinese population. However, there are several potential limitations that should be considered when interpreting the results. First, this is a cross-sectional study and no causal relationship can be established. Further follow-up of the population and more prospective studies will be needed to validate our findings. Secondly, some variables such as physical activity and dietary patterns which might affect renal function were not included in our study. Finally, the gold standards for measurement of fat distribution are micromagnetic resonance imaging and microcomputed tomography [31]; in our investigation, we used the Omron body composition monitor, which may be less accurate than gold standards. However, the Omron device has been used in various studies and has demonstrated reasonable accuracy $[32,33]$.

\section{Conclusion}

Obesity was associated with an increased risk of CKD, with PBF being the best adiposity index predictor for identifying CKD. Given that obesity is modifiable risk factor, CKD patients should manage their weight to prevent CKD progression. Patients should also be screened for high PBF in CKD prevention efforts.

\section{Additional files}

Additional file 1: List of investigators of the China Hypertension Survey Study. (DOCX $14 \mathrm{~kb}$ )

Additional file 2: List of IRBs of sub-centers. (DOCX $13 \mathrm{~kb}$ )
Abbreviations

AUC: Area under the ROC curve; BMI: Body mass index; CKD: Chronic kidney disease; eGFR: Estimated glomerular filtration rate; PBF: Percentage body fat; ROC curve: Receiver operating characteristics curve; VFI: Visceral fat index; WC: Waist circumference; WHtR: Waist-to-height ratio

\section{Acknowledgements}

The survey could be accomplished through the fine work of the staff in the national level, and we thank for all the colleagues involving in the survey (Additional file 1). We gratefully acknowledge Tianming Zhao, Guohui Fan, Jingyu Nie, Suning Li for help in maintaining the data, and Dominique Comer Medaglio, Zugui Zhang (from Christiana Care Health System, USA) for help in improving the manuscript.

The authors are grateful to OMRON Corporation, Kyoto, Japan, for supporting Blood Pressure Monitor (HBP-1300) and body fat measurement device (V-body HBF-371); Henan Medical Science \& Technology Co., Ltd., China, for Digital ECG device (GY-5000); Microlife, Taipei, China, for Automated ABI device (Watch BP Office device).

\section{Funding}

This work was supported by a grant from ministry of Science \& Technology, China (No.:2011BAl11B01); from National Health and Family Planning Commission, China (No.201402002) and from BUCHANG PHARMA, Xian, China, Pfizer China, and Essen Technology Company Limited.

All funders had no role in study design, data collection and analysis, decision to publish, or preparation of the manuscript.

\section{Availability of data and materials}

The datasets used and/or analyzed during the current study are available from the corresponding author on reasonable request.

Authors' contributions

$Z W, Z C, X W, L Z, L S, Y T$ and $R G$ participated in the design of the study $Z W, Z C, X W, L Z, J N, C Z, J W, L S, Y T$ and $R G$, participated in the data collection; YD and ZC performed the statistical analysis; YD wrote the manuscript, ZW supervised and made critical revisions of the manuscript. All authors have read and approved the final manuscript. 


\section{Ethics approval and consent to participate}

The ethical review committee of Fuwai Hospital (Beijing, China) and each participating center (Additional file 2) approved the study's protocol. And all the participants gave written informed consent prior to data collection.

\section{Consent for publication}

Not applicable

\section{Competing interests}

The authors declare that they have no competing interests from BUCHANG PHARMA, Xian, China, Pfizer China, and Essen Technology Company Limited. All the funders had no role in study design, data collection and analysis, decision to publish, or preparation of the manuscript.

\section{Publisher's Note}

Springer Nature remains neutral with regard to jurisdictional claims in published maps and institutional affiliations.

\section{Author details}

'Division of Prevention and Community Health, National Center for Cardiovascular Disease, Fuwai Hospital, Pecking Union Medical College \& Chinese Academy of Medical Sciences, Beijing 102308, China. ${ }^{2}$ Fuwai Hospital, Pecking Union Medical College \& Chinese Academy of Medical Sciences, Beijing 100037, China.

Received: 2 August 2017 Accepted: 7 February 2018 Published online: 17 February 2018

\section{References}

1. Keith DS, Nichols GA, Gullion CM, Brown JB, Smith DH. Longitudinal followup and outcomes among a population with chronic kidney disease in a large managed care organization. Arch Intern Med. 2004;164(6):659-63.

2. Go AS, Chertow GM, Fan D, McCulloch CE, Hsu CY. Chronic kidney disease and the risks of death, cardiovascular events, and hospitalization. N Engl J Med. 2004;351(13):1296-305.

3. Smyth A, Glynn LG, Murphy AW, Mulqueen J, Canavan M, Reddan DN, O'Donnell M. Mild chronic kidney disease and functional impairment in community-dwelling older adults. Age Ageing. 2013; 42(4):488-94.

4. Codreanu I, Perico N, Sharma SK, Schieppati A, Remuzzi G. Prevention programmes of progressive renal disease in developing nations. Nephrology (Carlton). 2006;11(4):321-8.

5. McClellan WM, Abramson J, Newsome B, Temple E, Wadley VG, Audhya P, McClure LA, Howard VJ, Warnock DG, Kimmel P. Physical and psychological burden of chronic kidney disease among older adults. Am J Nephrol. 2010; 31(4):309-17.

6. Zhang L, Wang F, Wang L, Wang W, Liu B, Liu J, Chen M, He Q, Liao Y, Yu X, et al. Prevalence of chronic kidney disease in China: a cross-sectional survey. Lancet. 2012;379(9818):815-22.

7. Hu L, Huang X, You C, Li J, Hong K, Li P, Wu Y, Wu Q, Wang Z, Gao R, et al. Prevalence of overweight, obesity, abdominal obesity and obesity-related risk factors in southern China. PLoS One. 2017;12(9):e0183934.

8. Xi B, Liang Y, He T, Reilly KH, Hu Y, Wang Q, Yan Y, Mi J. Secular trends in the prevalence of general and abdominal obesity among Chinese adults, 1993-2009. Obes Rev. 2012;13(3):287-96.

9. Kovesdy CP, Furth S, Zoccali C. Obesity and kidney disease: hidden consequences of the epidemic. Clin Kidney J. 2017;10(1):1-8.

10. Zeng Q, He Y, Dong S, Zhao X, Chen Z, Song Z, Chang G, Yang F, Wang Y. Optimal cut-off values of BMl, waist circumference and waist:height ratio for defining obesity in Chinese adults. Br J Nutr. 2014;112(10):1735-44.

11. Jiang J, Deng S, Chen Y, Liang S, Ma N, Xu Y, Chen X, Cao X, Song C, Nie W, et al. Comparison of visceral and body fat indices and anthropometric measures in relation to untreated hypertension by age and gender among Chinese. Int J Cardiol. 2016;219:204-11.

12. Zeng Q, Dong SY, Sun XN, Xie J, Cui Y. Percent body fat is a better predictor of cardiovascular risk factors than body mass index. Braz J Med Biol Res. 2012;45(7):591-600.

13. Wang Z, Zhang L, Chen Z, Wang X, Shao L, Guo M, Zhu M, Gao R. Survey on prevalence of hypertension in China: background, aim, method and design. Int J Cardiol. 2014;174(3):721-3.
14. Ma YC, Zuo L, Chen JH, Luo Q, Yu XQ, Li Y, Xu JS, Huang SM, Wang LN, Huang $W$, et al. Modified glomerular filtration rate estimating equation for Chinese patients with chronic kidney disease. J Am Soc Nephrol. 2006; 17(10):2937-44.

15. DeLong ER, DeLong DM, Clarke-Pearson DL. Comparing the areas under two or more correlated receiver operating characteristic curves: a nonparametric approach. Biometrics. 1988;44(3):837-45.

16. Chen W, Chen W, Wang H, Dong X, Liu Q, Mao H, Tan J, Lin J, Zhou F, Luo $\mathrm{N}$, et al. Prevalence and risk factors associated with chronic kidney disease in an adult population from southern China. Nephrol Dial Transplant. 2009; 24(4):1205-12.

17. Zhang L, Zuo L, Xu G, Wang F, Wang M, Wang S, Lv J, Liu L, Wang H. Community-based screening for chronic kidney disease among populations older than 40 years in Beijing. Nephrol Dial Transplant. 2007;22(4):1093-9.

18. Lin B, Shao L, Luo Q, Ou-yang L, Zhou F, Du B, He Q, Wu J, Xu N, Chen J. Prevalence of chronic kidney disease and its association with metabolic diseases: a cross-sectional survey in Zhejiang province, Eastern China. BMC Nephrol. 2014;15(1):36-42. doi:https://doi.org/10.1186/1471-2369-15-36.

19. He Y, Liu D, Tan W, Ma X, Lian F, Xu X. Association between body mass index and mildly decreased estimated glomerular filtration rate in Chinese adults with early chronic kidney disease. J Ren Nutr. 2016;26(6):367-72.

20. Lew QJ, Jafar TH, Talaei M, Jin A, Chow KY, Yuan JM, Koh WP. Increased body mass index is a risk factor for end-stage renal disease in the Chinese Singapore population. Kidney Int. 2017;92(4):979-87.

21. Noori N, Hosseinpanah F, Nasiri AA, Azizi F. Comparison of overall obesity and abdominal adiposity in predicting chronic kidney disease incidence among adults. J Ren Nutr. 2009;19(3):228-37.

22. Muller MJ, Lagerpusch M, Enderle J, Schautz B, Heller M, Bosy-Westphal A. Beyond the body mass index: tracking body composition in the pathogenesis of obesity and the metabolic syndrome. Obes Rev. 2012;13(Suppl 2):6-13.

23. Jaroszynski A, Derezinski T, Jaroszynska A, Zapolski T, Wasikowska B, Wysokinski A, Jawien A, Zaluska W, Horoch A. Association of anthropometric measures of obesity and chronic kidney disease in elderly women. Ann Agric Environ Med. 2016;23(4):636-40

24. Dai D, Chang Y, Chen Y, Chen S, Yu S, Guo X, Sun Y. Visceral adiposity index and lipid accumulation product index: two alternate body indices to identify chronic kidney disease among the rural population in Northeast China. Int J Environ Res Public Health. 2016;13(12):1231-41.

25. Yang S, Xiao F, Pan L, Zhang H, Ma Z, Liu S, Liu Y, Zhang W, Zeng X, Liu C, et al. Association of serum irisin and body composition with chronic kidney disease in obese Chinese adults: a cross-sectional study. BMC Nephrol. 2015; 16:16-26. doi:https://doi.org/10.1186/s12882-015-0009-5.

26. Soltani Z, Washco V, Morse S, Reisin E. The impacts of obesity on the cardiovascular and renal systems: cascade of events and therapeutic approaches. Curr Hypertens Rep. 2015;17(2):1-14. doi:https://doi.org/10. 1007/s11906-014-0520-2.

27. Snyder S, Turner GA, Turner A. Obesity-related kidney disease. Prim Care. 2014;41(4):875-93

28. Zhang XL, Topley $\mathrm{N}$, Ito T, Phillips A. Interleukin-6 regulation of transforming growth factor (TGF)-beta receptor compartmentalization and turnover enhances TGF-beta1 signaling. J Biol Chem. 2005;280(13):12239-45.

29. Takano Y, Yamauchi K, Hayakawa K, Hiramatsu N, Kasai A, Okamura M, Yokouchi M, Shitamura A, Yao J, Kitamura M. Transcriptional suppression of nephrin in podocytes by macrophages: roles of inflammatory cytokines and involvement of the PI3KJAkt pathway. FEBS Lett. 2007;581(3):421-6.

30. Ikezumi Y, Suzuki T, Karasawa T, Kawachi H, Nikolic-Paterson DJ, Uchiyama M. Activated macrophages down-regulate podocyte nephrin and podocin expression via stress-activated protein kinases. Biochem Biophys Res Commun. 2008;376(4):706-11.

31. Chen W, Wilson JL, Khaksari M, Cowley MA, Enriori PJ. Abdominal fat analyzed by DEXA scan reflects visceral body fat and improves the phenotype description and the assessment of metabolic risk in mice. Am J Physiol Endocrinol Metab. 2012;303(5):E635-43.

32. Ballesteros-Pomar MD, Calleja-Fernandez A, Diez-Rodriguez R, VidalCasariego A, Blanco-Suarez MD, Cano-Rodriguez I. Comparison of different body composition measurements in severely obese patients in the clinical setting. Nutr Hosp. 2012;27(5):1626-30.

33. Martin Moreno V, Gomez Gandoy B, Antoranz Gonzalez M, Fernandez Herranz S, Gomez De La Camara A, de Oya Otero M. Validation of the OMRON BF 300 monitor for measuring body fat by bioelectric impedance. Aten Primaria. 2001;28(3):174-81. 